\title{
Asthma management in a public referral center in Porto Alegre in comparison with the guidelines established in the 111 Brazilian Consensus on Asthma Management*
}

\author{
WALDO MATTOS', LUCIANO BAUER GROHS², FABÍOLA ROQUE ${ }^{3}$, \\ MAURÍCIO FERREIRA ${ }^{3}$, GABRIELA MÂNICA ${ }^{3}$, ERNESTO SOARES ${ }^{3}$
}

\begin{abstract}
Objective: To determine whether the guidelines put forth in the III Brazilian Consensus on Asthma Management are being applied in a population of asthma patients treated at a public hospital that is a referral center for asthma in the city of Porto Alegre, Brazil. Methods: All adult asthma patients who began their treatment between 1999 and 2002 were evaluated. The treatment given was classified as consistent or inconsistent with the Consensus guidelines. The clinical features of asthma and the frequency of treatment provided by a specialist were compared between the two groups (those receiving guideline-consistent treatment and those receiving guideline-inconsistent treatment). Results: The charts of 357 patients were evaluated. The study sample consisted of 106 males (29.9\%) and 251 females (70.3\%). The mean age was 41 years, and 33 (9.2\%) of the patients were smokers. The treatment was considered inconsistent with the Consensus guidelines in 246 cases (70\%). Of those 246, 174 (71\%) had presented persistent asthma and were not treated with an inhaled corticosteroid. Normal forced expiratory volume in one second, being from 12 to 18 years of age, and having intermittent asthma were more frequently observed among the patients receiving guideline-consistent treatment ( $p<0.01)$. No correlations were found between guideline-inconsistent treatment and being treated by a pulmonologist, severity of persistent asthma or number of emergency room visits. Conclusion: Most of the asthma patients treated at the public referral center in Porto Alegre did not receive treatment that was consistent with the Consensus guidelines. Undertreatment with inhaled corticosteroids was the principal source of that inconsistency.
\end{abstract}

Keywords: Asthma/therapy; Guidelines; Comparative study

\footnotetext{
* Study carried out at the Fundação Faculdade Federal de Ciências Médicas de Porto Alegre (FFFCMPA, Federal Foundation School of Medical Sciences of Porto Alegre) - Porto Alegre, Rio Grande do Sul, Brazil.

1. Adjunct Professor in the Internal Medicine Department of the Fundação Faculdade Federal de Ciências Médicas de Porto Alegre (FFFCMPA, Federal Foundation School of Medical Sciences of Porto Alegre); Chief Resident in Internal Medicine at the Hospital Nossa Senhora da Conceição - Porto Alegre, Rio Grande do Sul, Brazil.

2. Medical resident in Pulmonology at the Hospital Nossa Senhora da Conceição - Porto Alegre, Rio Grande do Sul, Brazil. 3. Medical student at the Fundação Faculdade Federal de Ciências Médicas de Porto Alegre (FFFCMPA, Federal Foundation School of Medical Sciences of Porto Alegre) - Porto Alegre, Rio Grande do Sul, Brazil.

Correspondence to: Waldo Mattos. Rua Santana, 1.253, sala 206 - CEP: 90040-373, Porto Alegre, RS, Brasil.

Tel: 5551 3219-6664. Email: waldomattos@uol.com.br

Submitted: 16 January 2005. Accepted, after review: 29 November 2005.
} 


\section{INTRODUCTION}

It is estimated that there are approximately ten million individuals with asthma in Brazil. 1ts negative impact on the population includes the economic costs of the health system structures and the use of medications, as well as the harmful effects on various aspects of patient health, from compromised quality of life to death. In 2001, the Hospital Information Service of the Ministry of Health registered 365,966 hospitalizations and 2454 deaths from asthma, at a cost of $\mathrm{R} \$ 107,123,187.75$ (in Brazilian reals), considering only the transfer of the value stipulated for authorizations for hospital admission. ${ }^{(1)}$ These data symbolize the concept that this disease is a true public health problem.

Many factors have been identified as possible causes of asthma morbidity and mortality: patient lack of understanding of the disease; inadequate medical prescriptions; low compliance with treatment; inability to use inhalation; absence of a written plan to face exacerbations; and limited use of functional evaluation in the diagnosis and staging of asthma severity. ${ }^{(2-13)}$

In the attempt to improve asthma control, consensus guidelines including all of the relevant scientific information published have been systematically drawn up in various countries in order to aid health professionals in carrying out the treatment of these patients. However, the use of these guidelines by health professionals, in rich as well as in poor countries, have proven quite insufficient. ${ }^{(14-17)}$ This inadequacy has been reported to occur in the treatment of the exacerbation as well as in the period between asthma attacks. In one study, ${ }^{(18)}$ it was demonstrated that $62 \%$ of asthma patients treated in the emergency room were undertreated, $87 \%$ had no written plan of action for emergency situations, and 73\% did not know how to measure peak expiratory flow (PEF). Similarly, other authors have reported that only 54\% of the patients with severe asthma used an inhaled corticosteroid as part of their daily outpatient treatment.

One component of this problem that remains relevant is the lack of knowledge on the part of health professionals. In the USA, medical knowledge regarding the content provided by the National Heart Lung and Blood Institute was assessed through the use of a questionnaire, and the mean total proportion of correct responses, among specialists and nonspecialists, was found to be only $60 \% .^{(17)}$ Some authors have reported that the training of resident physicians improves aspects of asthma treatment but not of its diagnosis. ${ }^{(19)}$

In Brazil, three consensus guidelines on asthma management have been established, the most recent one in 2002. However, it remains unclear how much these documents have helped to change the panorama of asthma control in the country. The objective of the present study was to determine whether the guidelines put forth in the 111 Brazilian Consensus on Asthma Management are being applied, in clinical practice, in a population of patients served by the public health system in the city of Porto Alegre, Brazil. ${ }^{(20)}$

\section{METHODS}

We evaluated, retrospectively, the information collected during the first medical appointment and contained in the charts of patients referred to the Asthma Clinic of the Pulmonology Department of the Nossa Senhora da Conceição Hospital (a teaching hospital with ties to the Ministry of Health) between 1999 and 2002. All of the charts evaluated were for patients who had been diagnosed with asthma in the first medical appointment or during the follow-up treatment.

The diagnostic criteria were as follows: presenting symptoms (dyspnea, chronic cough, or wheezing); having obstructive ventilatory disorder, which was defined as forced expiratory volume in one second $\left(\mathrm{FEV}_{1}\right)<80 \%$ of predicted and ratio between $\mathrm{FEV}_{1}$ and forced vital capacity $<0.7$. Patients meeting these criteria in the initial evaluation or during the follow-up treatment were classified as asthma sufferers. Patients presenting chronic obstructive pulmonary disease, bronchiectasis, tuberculosis sequelae, or any other chronic pulmonary disease were excluded. The medications that the patients themselves reported taking in the final evaluation were considered to constitute the treatment regimen at the moment of the first medical appointment, and this treatment regimen was compared with that recommended in the guidelines established in the 111 Brazilian Consensus on Asthma Management. Patients having had no medical appointments within the last twelve months were not considered to be in follow-up treatment.

The data reviewed were as follows: gender; age; history of smoking; symptoms; spirometry or PEF results; medication used; specialization of the physician who treated the patient previously; and 
number of emergency room visits within the twelve months prior to and after the medical appointment. The clinical form filled out in the first evaluation is standardized, and all the information regarding the variables mentioned above was thereby registered at the moment of the interview, except for the number of emergency room visits, which was obtained prospectively through the computerized system of registration of medical appointments. The system records the date and location (for example, emergency room) of all visits but does not include the reason for the medical appointment.

All patients were submitted to blood workups. In addition, total $\lg$ E serum levels were determined, PEF was measured, spirometry (including a pharmacodynamic test) was performed, and X-rays (of the chest and sinuses) were taken. Asthma was classified as intermittent or persistent and was categorized as mild, moderate, or severe according to the guidelines established in the 111 Brazilian Consensus on Asthma Management, ${ }^{(20)}$ which are based on the symptoms, the impact on patient activities, the intensity of exacerbations, the presence of nocturnal symptoms, the use of relief medication, the PEF, and the FEV .

The treatment was then classified as consistent or inconsistent with the guidelines established in the 111 Brazilian Consensus on Asthma Management ${ }^{(20)}$ : intermittent asthma - use of a short-acting $\beta 2$ agonist alone; mild persistent asthma - use of low doses of an inhaled corticosteroid (400 g/day of beclomethasone or equivalent) or antileukotriene; moderate persistent asthma - use of low or moderate doses of an inhaled corticosteroid (ranging from 400 to $800 \mathrm{~g} /$ day of beclomethasone or equivalent), in combination with a long-acting $\beta 2$ agonist or high doses of an inhaled corticosteroid (> $800 \mathrm{~g} /$ day of beclomethasone or equivalent); severe persistent asthma - use of high doses of an inhaled corticosteroid, a long-acting $\beta 2$ agonist, an oral corticosteroid, theophylline, or antileukotriene drugs.

The data were organized using Microsoft Excel, version 2000, and were analyzed using the SPSS program, version 10.2. The analysis of variance Ftest or the Student's t-test was used in the analysis of quantitative variables, whereas the chi-square test was used to analyze categorical variables. The study of some quantitative variables was complemented by a correlation analysis using the Spearman correlation coefficient. A 95\% confidence interval was adopted, and values of $p<0.05$ were considered statistically significant.

\section{RESULTS}

The medical charts of 357 patients, of which 106 (29.7\%) were male and 251 (70.3\%) were female, were analyzed (Table 1). Ages ranged from 11 to 80 years (mean, $41 \pm 18$ years). Of those 357 patients, 287 (80.4\%) were Caucasian, and 70 (19.6\%) were non-white. In addition, 33 (9.2\%) were smokers, and 97 (27.2\%) were former smokers. A total of 4 patients were excluded from the analysis due to lack of information in their charts.

In the initial medical appointment, the treatment reported was consistent with what is recommended in the Brazilian Consensus guidelines according to asthma severity in 107 patients (30\%) and inconsistent in the remaining 246 (70\%). Treatments were considered guideline-consistent in 55/71 cases of intermittent asthma (78\%), 22/106 cases of mild persistent asthma (20\%), 12/113 cases of moderate persistent asthma (11\%), and 31/63 cases of severe persistent asthma (49\%). The percentage of patients with intermittent asthma receiving guidelineconsistent treatment was greater than that observed

TABLE 1

Characteristics of the patients studied

\begin{tabular}{lrr}
\hline & $\mathrm{n}$ & \multicolumn{1}{l}{$(\%)$} \\
\hline Gender & & \\
$\quad$ Male & 106 & $(29.7 \%)$ \\
$\quad$ Female & 251 & $(70.3 \%)$ \\
Race & & \\
$\quad$ White & 287 & $(80.4 \%)$ \\
$\quad$ Black & 70 & $(19.6 \%)$ \\
Smoking, n (\%) & & \\
$\quad$ Smokers & 33 & $(9.2 \%)$ \\
Former smokers & 97 & $(27.2 \%)$ \\
Non-smokers & 175 & $(49 \%)$ \\
$\quad$ No information & 52 & $(14.6 \%)$ \\
Age, n (\%) & & \\
$\quad<12$ years & 10 & $(2.8 \%)$ \\
12-18 years & 38 & $(10.6 \%)$ \\
18-40 years & 122 & $(34.2 \%)$ \\
$40-65$ years & 147 & $(41.2 \%)$ \\
$>65$ years & 40 & $(11.2 \%)$ \\
Asthma severity & & \\
IA & 71 & $(19.9 \%)$ \\
MPA & 106 & $(29.7 \%)$ \\
MoPA & 113 & $(31.7 \%)$ \\
SPA & 63 & $(17.6 \%)$ \\
No classification & 4 & $(1.1 \%)$ \\
\hline IA intermittent asthma & &
\end{tabular}

IA: intermittent asthma; MPA: mild persistent asthma; MoPA: moderate persistent asthma; SPA: severe persistent asthma. 
among patients with persistent asthma $(p<0.0001)$.

There were no statistically significant differences among the degrees of persistent asthma (Figure 1). However, we detected a tendency toward a smaller number of guideline-inconsistent treatments among the cases of severe persistent asthma.

Of the 246 patients receiving guidelineinconsistent treatment, 174 (71\%) were patients with persistent asthma who were treated with a corticosteroid, 43 (17\%) were patients for whom the use of an oral corticosteroid was not considered necessary, 17 (7\%) were patients with intermittent asthma who were under treatment with a corticosteroid, and $12(5 \%)$ were patients with persistent asthma treated with doses of an inhaled corticosteroid that were lower than those recommended.

The frequency of guideline-consistent treatments was found to be the highest among the youngest patients (those between 12 and 18 years of age) and among those presenting normal FEV in the initial spirometric test $(p<0.01)$. The treatments received by the patients at the moment of the initial evaluation are described in Table 2 . In addition, we observed a higher prevalence of intermittent asthma and mild persistent asthma among the young patients $(\mathrm{p}=$ 0.01 ). Figure 2 shows the age distribution in the groups of patients receiving guideline-consistent and guideline-inconsistent treatment.

There was a slight tendency, with no statistical significance, toward a greater number of guidelineinconsistent treatments among the patients previously treated by non-pulmonologists (Figure 3). The

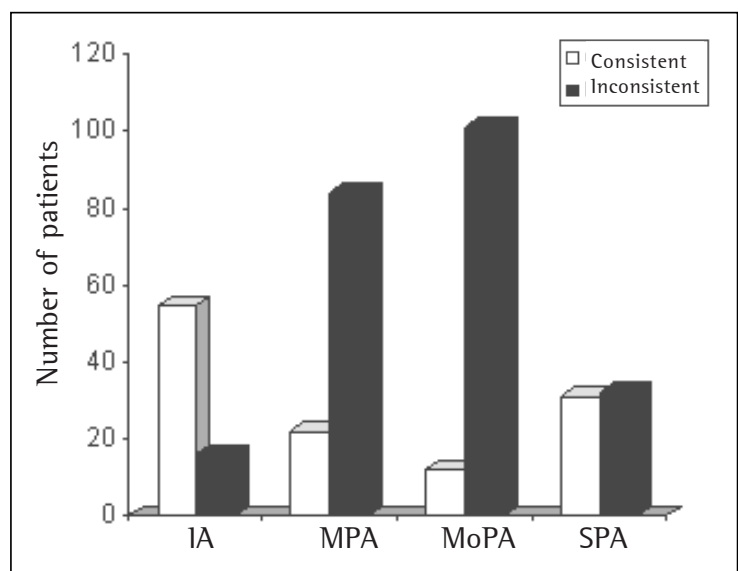

Figure 1 - Guideline-consistency of the treatment according to asthma severity. IA: intermittent asthma; MPA: mild persistent asthma; MoPA: moderate persistent asthma; SPA: severe persistent asthma. ${ }^{*} p<0.0001$.
TABLE 2

Characteristics of the drug therapy in the initial evaluation

\begin{tabular}{lrrrr}
\hline & IA & MPA & MoPA & SPA \\
Number of patients & \multicolumn{1}{c}{71} & \multicolumn{1}{c}{106} & \multicolumn{1}{c}{113} & \multicolumn{1}{c}{63} \\
\hline No treatment & $26(37 \%)$ & $25(24 \%)$ & $15(13 \%)$ & $6(9 \%)$ \\
Short-acting $\beta 2$ & $46(65 \%)$ & $73(69 \%)$ & $86(76 \%)$ & $52(82 \%)$ \\
Long-acting $\beta 2$ & 0 & 0 & 0 & 0 \\
Ipratropium bromide & 0 & $1(1 \%)$ & $1(1 \%)$ & $2(3 \%)$ \\
Xanthine & $11(15 \%)$ & $2(2 \%)$ & $30(26 \%)$ & $19(30 \%)$ \\
lnhaled corticosteroid & $14(20 \%)$ & $22(21 \%)$ & $12(11 \%)$ & $12(19 \%)$ \\
Oral corticosteroid & $3(4 \%)$ & $17(16 \%)$ & $26(23 \%)$ & $19(30 \%)$ \\
\hline
\end{tabular}

IA: intermittent asthma; MPA: mild persistent asthma; MoPA: moderate persistent asthma; SPA: severe persistent asthma.

treatment was considered guideline-inconsistent in $21 / 31$ cases being treated by pulmonologists (68\%), in $105 / 140$ cases being treated by any other physicians other than pulmonologists (75\%), and in 93/122 cases of patients not in follow-treatment (76\%). In 64 cases, it was not possible to categorize the treatment based on the information contained in the medical charts.

The number of emergency room visits within the last twelve months prior to the medical appointment was similar among the patients treated by pulmonologists and those treated by nonpulmonologists $(2.84 \pm 2.05$ vs. $2.96 \pm 3.53$ visits, respectively). There were no statistically significant differences between the twelve months preceding the initial evaluation and the twelve subsequent months in terms of the total number of emergency room visits ( $2.97 \pm 4.12$ vs. $2.56 \pm 5.87)$. In addition, we observed a positive correlation between the pre-

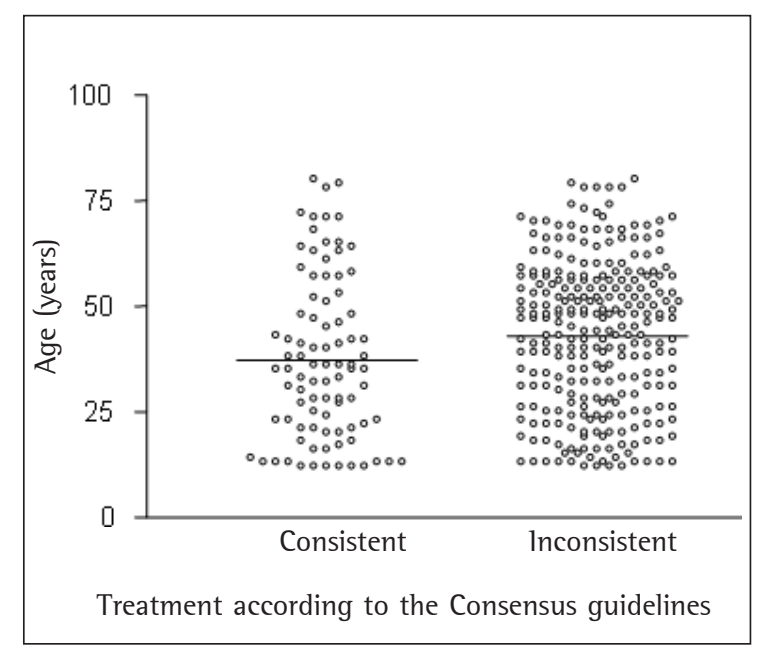

Figure 2 - Guideline-consistency of the treatment according to age 


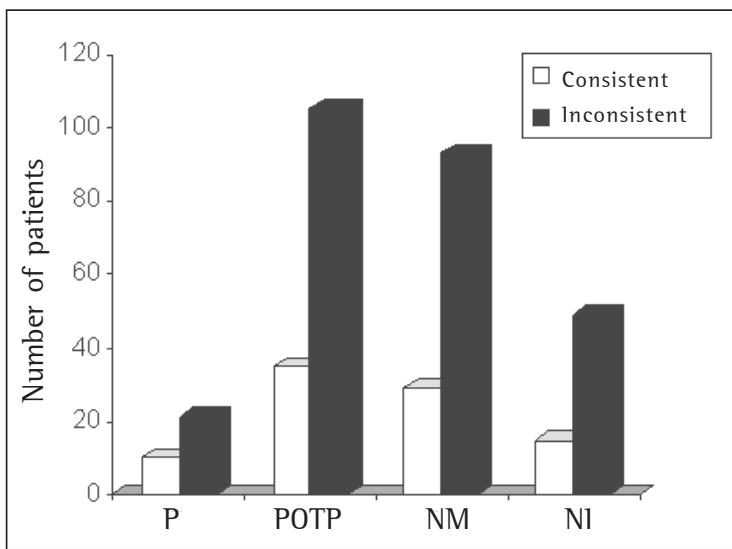

Figure 3 - Monitoring by pulmonologists and guidelineconsistency of the treatment

P: pulmonologist; POTP: physician other than a pulmonologist: NM: no monitoring; Nl: no information.

evaluation and post-evaluation periods in patients who had visited the emergency room at least once in both periods $(r=0.65, p<0.0001)$. The number of emergency room visits was not found to correlate with asthma severity at the time of the initial evaluation or with underutilization of inhaled corticosteroids.

\section{DISCUSSION}

In the present study, it was demonstrated that most of the public heath care system asthma patients who were first treated at the referral center in Porto Alegre did not receive treatment consistent with the Consensus guidelines, and that underutilization of inhaled corticosteroids was the greatest problem. In the subgroup of patients with intermittent asthma, for whom the use of inhaled corticosteroids is not recommended in the Consensus guidelines, the treatment was most often in keeping with the guidelines.

These findings must be interpreted with caution, and many causal factors must be considered. First, compliance with treatment among patients with persistent asthma does not differ from that seen among patients with other chronic diseases. Even in developed countries, where the access to the health system and medications is effectively possible, various reports in the literature indicate a rate of compliance of approximately 50\%..$^{(13,21-22)} \ln$ the present study, compliance with treatment was not evaluated, since we considered the treatment reported by the patient, and this does not necessarily imply that it was the treatment prescribed. It is possible that our results reflect a combination of these factors.

Second, we know that the lack of knowledge regarding the disease, principles of treatment, and procedures of use of inhaled medications is also considered another important determinant of treatment inconsistency. In fact, the inclusion of patients in asthma education programs, whose activities are based on information, promotes better asthma control by increasing compliance with the treatment regimens prescribed. Although there have been no Brazilian studies confirming the results obtained in NorthAmerican studies, ${ }^{(17)}$ the lack of knowledge on the part of physicians has also been considered a factor that contributes to treatment inconsistency.

We could presume that, since most of the patients analyzed in the present study were being treated by a general practitioner and had had no access to a formal education program about their disease, part of the problem might be the lack of awareness on the part of both the practitioner and the patient. However, we observed only a tendency, without statistical significance, toward higher frequency of guideline-consistent treatment among the patients previously treated by pulmonologists. The interpretation of this finding that the treatment provided by specialists, who are necessarily more upto-date with the treatment principles than are nonspecialists, did not result in a statistically significant difference is somewhat intriguing. A deeper understanding may depend on accepting the fact that an approach that does not guarantee unlimited access to the medication used to control the disease and prevent exacerbations, as well as potentially reducing the cost and the use of the public health care system infrastructure, as has been adopted by a number of health administrators, is the greatest of all failures. Therefore, it is possible that the limited availability of appropriate medications negates any advantage related to the better knowledge possessed by the specialist, resulting in no effective gain in the quality of the treatment.

In the present study, we also observed that the number of emergency room visits was not proportionally related to asthma severity or underutilization of corticosteroids, and that the patients who most frequently sought treatment in the emergency room prior to the asthma clinic evaluation continued to seek treatment in the emergency room after that specialized treatment. This may be a reflection of the problems inherent to our public health care system, in its current state, which is characterized by 
limited access (to physicians and to medication). We observed that there were a significant number of patients being treated unnecessarily with systemic corticosteroids, possibly only due to the fact that this medication is available in public health facilities, in contrast to inhaled corticosteroids, which are not readily available in health care services. These problems, taken together, probably explain why updating and disseminating the Consensus guidelines have lower effectiveness than expected.

The holding of regular consensus meetings and the regular publishing of consensus guidelines in national journals reinforce and expand the potential for dissemination of medical information. This has been done from the viewpoint that even the specialist can benefit from reviewing this information. However, we must bear in mind that most asthma patients are treated by non-specialists, and that, while the availability of drugs that control this disease remains restricted and access to outpatient treatment remains limited, no attenuation of this public health problem can be expected. Therefore, strategies to disseminate guidelines to the general practitioner may be more important.

\section{ACKNOWLEDGEMENTS}

The authors would like to thank Professor Viviane Mattos for designing the statistical evaluation.

\section{REFERENCES}

1. Brasil. Ministério da Saúde, Sistema de Informações Hospitalares do SUS (SIH-SUS) 2001 [texto na Internet]. Brasília: Ministério da Saúde; 2002. [citado 2005 Out 19]. Disponível em: http://www.datasus.gov.br/catalogo/ sihsus.htm.

2. O'Dowd LC, Fife D, Tenhave T, Panettieri RA Jr. Attitudes of physicians toward objective measures of airway function in asthma. Am J Med. 2003;114(5):391-6. Comment in: Am J Med. 2003;114(5):415-6.

3. Lukacs SL, France EK, Baron AE, Crane LA. Effectiveness of an asthma management program for pediatric members of a large health maintenance organization. Arch Pediatr Adolesc Med. 2002;156(9):872-6.

4. Forbis SG, Aligne CA. Poor readability of written asthma management plans found in national guidelines. Pediatrics. 2002;109(4):e52.

5. Cabana MD, Rand CS, Becher OJ, Rubin HR. Reasons for pediatrician nonadherence to asthma guidelines. Arch Pediatr Adolesc Med. 2001;155(9):1057-62.

6. Comino E, Henry R. Changing approaches to asthma management in Australia: effects on asthma morbidity. Drugs. 2001;61(9):1289-300.

7. Finkelstein JA, Lozano P, Shulruff R, Inui TS, Soumerai SB,
$\mathrm{Ng} \mathrm{M}$, et al. Self-reported physician practices for children with asthma: are national guidelines followed? Pediatrics. 2000;106(4 Suppl):886-96. Comment in: Pediatrics. 2000;106(4 Suppl):897-8.

8. Veninga CC, Lagerlov $\mathrm{P}$, Wahlstrom R, Muskova M, Denig P, Berkhof J, et al. Evaluating an educational intervention to improve the treatment of asthma in four European countries. Drug Education Project Group. Am J Respir Crit Care Med. 1999;160(4):1254-62.

9. Walsh LJ, Wong CA, Cooper S, Guhan AR, Pringle M, Tattersfield AE. Morbidity from asthma in relation to regular treatment: a community based study. Thorax. 1999;54(4):296-300.

10. Curry SJ. Organizational interventions to encourage guideline implementation. Chest. 2000;118(2 Suppl): 40-6.

11. Clark NM, Partridge MR. Strengthening asthma education to enhance disease control. Chest. 2002;121(5):1661-9.

12. Legorreta AP, Christian-Herman J, O'Connor RD, Hasan MM, Evans R, Leung KM. Compliance with national asthma management guidelines and specialty care: a health maintenance organization experience. Arch Intern Med. 1998;158(5):457-64. Comment in: Arch Intern Med. 1998;158(15):1720-3.

13. Milgrom H, Wamboldt F, Bender B. Monitoring adherence to the therapy of asthma. Curr Opin Allergy Clin Immunol. 2002;2(3):201-5.

14. Gottlieb DJ, Beiser AS, O'Connor GT. Poverty, race, and medication use are correlates of asthma hospitalization rates. A small area analysis in Boston. Chest. 1995;108(1):28-35.

15. Lang DM, Sherman MS, Polansky M. Guidelines and realities of asthma management. The Philadelphia story. Arch Intern Med. 1997;157(11):1193-200.

16. Vollmer WM, O'Hollaren M, Ettinger KM, Stibolt T, Wilkins J, Buist AS, et al. Specialty differences in the management of asthma. A cross-sectional assessment of allergists' patients and generalists' patients in a large HMO. Arch Intern Med. 1997;157(11):1201-8. Comment in: Arch Intern Med. 1998;158(5):542.

17. Doerschug KC, Peterson MW, Dayton CS, Kline JN. Asthma guidelines: an assessment of physician understanding and practice. Am J Respir Crit Care Med. 1999;159(6):1735-41. Comment in: Am J Respir Crit Care Med. 2000;161(1):330.

18. Taylor DM, Auble TE, Calhoun WJ, Mosesso VN Jr. Current outpatient management of asthma shows poor compliance with International Consensus Guidelines. Chest. 1999;116(6): 1638-45. Comment in: Chest. 1999;116(6):1509-10.

19. Vieira JE, Cukier A, Stelmach R, Kasahara DI, Gannam $S$, Warth M. Comparison of knowledge on asthma: doctors completing internal medicine residency and doctors completing medical school. Sao Paulo Med J. 2001;119(3):101-4.

20. Sociedade Brasileira de Pneumologia e Tisiologia. 111 Consenso Brasileiro no Manejo da Asma 2002. J Pneumol. 2002;28(Supl 1):S1-28.

21. Valenti WM Treatment adherence improves outcomes and manages costs. AIDS Read. 2001;11(2):77-80.

22. Bousquet J, Knani J, Henry C, Liard R, Richard A, Michel FB, et al. Undertreatment in a nonselected population of adult patients with asthma. J Allergy Clin Immunol. 1996;98(3): 514-21. Comment in: J Allergy Clin Immunol. 1998;101(4 Pt 1):570-1. 